\title{
The molecular disk surrounding the protostellar binary L1551 IRS5
}

\author{
C. V. M. Fridlund ${ }^{1}$, P. Bergman ${ }^{2}$, G. J. White ${ }^{3}$, G. L. Pilbratt ${ }^{1}$, and J. A. Tauber ${ }^{1}$ \\ 1 ESA Astrophysics Mission Division, Research and Scientific Support Department, ESTEC, PO Box 299, \\ 2200 AG Noordwijk, The Netherlands \\ 2 Centre for Astrophysics and Space Science at Chalmers University of Technology, Onsala Space Observatory, \\ 43992 Onsala, Sweden \\ 3 Unit for Space Sciences and Astronomy, Physics Department, University of Kent, Canterbury CT2 7NR, UK
}

Received 24 July 2001 / Accepted 23 October 2001

\begin{abstract}
The inner three arcminutes surrounding the Class 0/1 binary protostar L1551 IRS5 have been observed using the $J=1 \rightarrow 0$ transitions of the $\mathrm{HCO}^{+}, \mathrm{H}^{13} \mathrm{CO}^{+},{ }^{12} \mathrm{CO}$ and ${ }^{13} \mathrm{CO}$ molecular species. Since the line core of $\mathrm{HCO}^{+}$is self reversed over a substantial part of our map, observations of isotopomers such as $\mathrm{H}^{13} \mathrm{CO}^{+}$are required in order to estimate the mass of the molecular gas in the immediate vicinity of IRS5. Our observations demonstrate the presence of a large ( $\sim 7000$ AU radius) dense, possibly rotating, molecular disk with a mass of a few $M_{\odot}$ oriented perpendicular to the major axis of an extended molecular outflow. The disk is surrounded by an envelope with a radius of $\sim 10000 \mathrm{AU}$ that contains two massive (each $\sim 1 M_{\odot}$ ) clumps. One of these features appears to be kinematically disconnected from both the disk and the molecular outflow.
\end{abstract}

Key words. ISM: clouds - ISM: individual objects: L1551 - ISM: jets and outflows - stars: formation stars: pre-main sequence - radio lines: ISM

\section{Introduction}

Among bipolar molecular outflows, the one centered on the young stellar object IRS5 in L1551 was the first discovered (Snell et al. 1980). It is nearby -140 pc (Reipurth 1999) - allowing the study of small spatial elements, and oriented with its major outflow axis at a small angle $\left(\sim 15^{\circ}-20^{\circ}\right.$, Liseau \& Sandell 1986; Moneti et al. 1988; Rainey et al. 1987) with respect to the plane of the sky. This results in red- and blue-shifted outflow lobes which are spatially well separated on the sky. In combination with the recognition that bipolar molecular outflows play a central role in the star formation process, L1551 has been well studied over the last $\sim 20$ years (see e.g. Fridlund et al. 1997 for a review).

L1551 IRS5 has been classified as a low mass object (Fridlund et al. 1980), and shown to be double (Rodriguez et al. 1986; Bieging \& Cohen 1985; Looney et al. 1997; Fridlund \& Liseau 1998; Rodriguez et al. 1998). The two embedded protostellar objects are separated by $\sim 45 \mathrm{AU}$ $\left(\sim 0^{\prime \prime} \cdot 3\right)$, and obscured by up to $A_{V} \sim 150$ mag (e.g. Stocke et al. 1988; Smith et al. 1987; White et al. 2000). The structure responsible for this visual extinction is most

Send offprint requests to: C. V. M. Fridlund, e-mail: Malcolm.Fridlund@esa.int likely in the form of a dense dust and gas disk, as indicated by a number of studies (summarised in Table 1).

The aperture synthesis observations of Sargent et al. (1988) in $\mathrm{C}^{18} \mathrm{O}$, and the continuum (Woody et al. 1989) revealed a disk-like envelope, with an extension of roughly $700 \mathrm{AU}$, and a mass of $\sim 0.1 M_{\odot}$. Observing the continuum, Keene \& Masson (1990) reported a very dense object with a radius of $\sim 45 \mathrm{AU}$ and a mass of $0.6 M_{\odot}$ centered on L1551 IRS5. This condensed object appeared to be surrounded by an envelope with a radius of $\sim 1600 \mathrm{AU}$ and an estimated mass of $2 M_{\odot}-3 M_{\odot}$. A larger flattened structure with a diameter of $\sim 4000 \mathrm{AU}$ and a total mass of $\sim 0.3 M_{\odot}$ has been detected in interferometric observations in $\mathrm{H}^{13} \mathrm{CO}^{+}$(Saito et al. 1996). These authors find evidence for mass infall. A large molecular disk had previously been inferred from single dish observations of CS (Kaifu et al. 1984), albeit with a higher mass in the range of $1-5 M_{\odot}$ depending on the assumed geometry. The latter authors also suggested that the disk was rotating. Fridlund (1987) and Fridlund et al. (1989) suggested that a possible interpretation of their ${ }^{13} \mathrm{CO}$ observations was that a large $(\sim 5000-10000 \mathrm{AU})$ disk is rotating around IRS5. Mizuno et al. (1994) surveyed 15 protostellar cores, using $\mathrm{H}^{13} \mathrm{CO}^{+}$. For L1551 they arrive - under the assumption of a spherical, dynamically relaxed distribution 
Table 1. Summary of observations indicating a disk or dense envelope around the L1551 IRS5 binary.

\begin{tabular}{|c|c|c|c|c|}
\hline Study & $\begin{array}{l}\text { Size scale } \\
\mathrm{AU}\end{array}$ & $\begin{array}{l}\text { Mass } \\
M_{\odot}\end{array}$ & Molecule/Continuum & $\begin{array}{l}\text { Single Aperture/Interferometry } \\
\text { S/I }\end{array}$ \\
\hline Kaifu et al. (1984) & 20000 & $1-5$ & CS $(1-0)$ & $\mathrm{S}$ \\
\hline Fridlund et al. (1989) & $5000-10000$ & - & ${ }^{13} \mathrm{CO} J=1 \rightarrow 0$ & $\mathrm{~S}$ \\
\hline Sargent et al. (1988) & 1400 & 0.1 & $\mathrm{C}^{18} \mathrm{O} J=1 \rightarrow 0$ & I limited $(\mathrm{u}, \mathrm{v})$ sampling \\
\hline Woody et al. (1989) & $\lesssim 500$ & 0.08 & $1.4 \mathrm{~mm}$ & I limited $(\mathrm{u}, \mathrm{v})$ sampling \\
\hline Keene \& Masson (1990) & $\sim 45,1600$ & $0.6,2-3$ & $\mathrm{~mm}, \mathrm{~cm}$ & $\mathrm{I}$ \\
\hline Lay et al. (1994) & $\sim 200$ & $>0.02$ & $345 \mathrm{GHz}$ & I \\
\hline Mizuno et al. (1994) & $\sim 7000$ & $4.2^{1}$ & $\mathrm{H}^{13} \mathrm{CO}^{+} J=1 \rightarrow 0$ & $\mathrm{~S}$ \\
\hline Ladd et al. (1995) & 5000 & $\sim 1$ & $0.7-1 \mathrm{~mm}$ & $\mathrm{~S}$ \\
\hline Fuller et al. (1995) & $4000-6000$ & $\sim 0.2^{2}$ & $\mathrm{C}^{18} \mathrm{O} J=2 \rightarrow 1, \mathrm{C}^{17} \mathrm{O} J=2 \rightarrow 1$ & $\mathrm{~S}$ \\
\hline Ohashi et al. (1996) & $1200 \mathrm{AU}$ & 0.08 & ${ }^{13} \mathrm{CO} J=1 \rightarrow 0$ & I \\
\hline Saito et al. (1996) & $\sim 4000$ & 0.3 & $\mathrm{H}^{13} \mathrm{CO}^{+} J=1 \rightarrow 0$ & I \\
\hline Hogerheijde et al. (1997) & $\sim 5000$ & $0.1-0.3$ & $\mathrm{HCO}^{+}, \mathrm{H}^{13} \mathrm{CO}^{+}$, etc. & $\mathrm{S}, \mathrm{I}$ \\
\hline Hogerheijde et al. (1998) & $"$ & & & $"$ \\
\hline Momose et al. (1998) & $\sim 2500$ & 0.062 & $\mathrm{C}^{18} \mathrm{O} J=1 \rightarrow 0$ & I \\
\hline
\end{tabular}

- at a virial mass of $4.2 M_{\odot}$. Hogerheijde et al. (1997, 1998) have carried out mapping observations in a number of transitions (including $\mathrm{HCO}^{+}$and $\mathrm{H}^{13} \mathrm{CO}^{+}$) of several young embedded objects including L1551. A disk mass (within radius $\sim 2800 \mathrm{AU}$ ) of $0.1-0.3 M_{\odot}$ and a mass loss of $\sim 1.0 \times 10^{-4} M_{\odot} \mathrm{yr}^{-1}$ (both lobes) is indicated. Finally, Momose et al. (1998) used aperture synthesis observations of $\mathrm{C}^{18} \mathrm{O} J=1 \rightarrow 0$ to derive a mass of $0.062 M_{\odot}$ for a $2380 \times 1050$ AU central condensation.

The inner region $\left(r \lesssim 15^{\prime \prime}\right.$ equals $\lesssim 2000$ AU) have been observed with very high signal to noise in the ${ }^{12} \mathrm{CO}{ }^{13} \mathrm{CO}$ $\& \mathrm{HCO}^{+} J=1 \rightarrow 0$ molecular transitions (Fridlund \& Knee 1993). Their main result was - after concluding that the $\mathrm{HCO}^{+}$emission is optically thin in the line wings that the mass loss rate is $\dot{M} \gtrsim 1.0 \times 10^{-5} M_{\odot} \mathrm{yr}^{-1}$. This value of $\dot{M}$ is consistent with that found from the average properties of the complete outflow indicating that the "current" $\dot{M}$ is representative for the whole dynamical life time of the outflow ( $\sim 10^{5}$ yrs).

Because of the apparent disparity of the results for the sizes and masses of the structures surrounding L1551 IRS5, we have extended on previous work by carrying out:

- Single-dish, high spectral resolution, high signal/noise molecular line observations in order to verify the presence of a (molecular) disk like structure;

- Determination of the disk properties;

- Observation of the interfaces between this disk like structure and the surrounding envelope/outflow through multi-species and isotopomer observations.

\section{Observations and data reduction}

We observed transitions of ${ }^{12} \mathrm{CO},{ }^{13} \mathrm{CO}, \mathrm{HCO}^{+}$and $\mathrm{H}^{13} \mathrm{CO}^{+}$, during two observing runs in March 1998 and June 2000, using the Onsala Space Observatory (OSO) $20 \mathrm{~m}$ telescope located at Onsala, Sweden. Data for the observations can be found in Table 2. The SIS SSB tuned receiver temperature was $\sim 100 \mathrm{~K}$ for all transitions utilised. The signal was fed into two backends. The multichannel filterbank backend used have 256 channels each with $250 \mathrm{kHz}$ resolution. An autocorrelator backend was used in parallel. This was configured to have 1600 channels with a channel separation of $25 \mathrm{kHz}$. We mainly used data obtained with the latter, using the former as an independent check on the quality of our observations. Atmospheric calibration was carried out using the chopper wheel method (Kutner \& Ulich 1981). The forward scattering efficiency, $\eta_{\mathrm{fss}}$, is estimated to be $0.70-0.57$ at

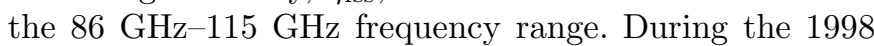
run, the observations were carried out under very good weather conditions resulting in SSB temperatures on the sky (above the atmosphere) of between $200 \mathrm{~K}$ and $400 \mathrm{~K}$ for $\mathrm{H}^{13} \mathrm{CO}^{+}, \mathrm{HCO}^{+}$and ${ }^{13} \mathrm{CO}$, with higher values of $\lesssim 1000 \mathrm{~K}$ for ${ }^{12} \mathrm{CO}$. In 2000 conditions were a little worse with system noise temperatures of $\lesssim 600 \mathrm{~K}$. This run was only used to expand the map of $\mathrm{H}^{13} \mathrm{CO}^{+}$, and the lower quality of the data had no significant impact on our interpretation. All observations were obtained using frequency switching, and reduced in the standard fashion (folding, removal of low-order polynomials from the baselines and scaling with $\eta_{\mathrm{fss}}$ ) resulting in spectra with a $T_{\mathrm{R}}^{*}$ temperature scale. All data have been reduced using the XSpackage written by P. Bergman. Maps around the position of IRS5 were made according to Table 2 . All offset positions in this paper are with respect to the IRS5 position.

\section{Observational results}

\section{1. $\mathrm{HCO}^{+}$and $\mathrm{H}^{13} \mathrm{CO}^{+}$}

In Fig. 1 our $\mathrm{H}^{13} \mathrm{CO}^{+}$spectra are displayed superposed on top of the corresponding $\mathrm{HCO}^{+}$spectra. A visual inspection of the data show a doubled peaked structure of 
Table 2. Observing parameters for the observations carried out in 1998 and 2000 . The reference $\left(0^{\prime \prime}, 0^{\prime \prime}\right)$ position is coincident with IRS5 $\left(\alpha=4^{\mathrm{h}} 28^{\mathrm{m}} 40^{\mathrm{s}}, \delta=18^{\circ} 01^{\prime} 42^{\prime \prime}\right.$, epoch 1950). $v_{\mathrm{LSR}}=6.3 \mathrm{~km} \mathrm{~s}^{-1}$ for L1551 The higher noise for $\mathrm{H}^{13} \mathrm{CO}^{+}$refers to the data obtained in 2000 and is readily identified in Fig. 1.

\begin{tabular}{llllllll}
\hline $\begin{array}{l}\text { Isotopic } \\
\text { species }\end{array}$ & Transition & $\begin{array}{l}\text { Frequency } \\
(\mathrm{GHz})\end{array}$ & $\begin{array}{l}\text { Map } \\
\text { size }\end{array}$ & $\begin{array}{l}\text { Beam } \\
\text { size }\left(^{\prime \prime}\right)\end{array}$ & $\begin{array}{l}\text { Sampling } \\
\text { step }\left(^{\prime \prime}\right)\end{array}$ & $\begin{array}{l}\text { Velocity } \\
\text { resolution m s }{ }^{-1}\end{array}$ & $\begin{array}{l}\text { RMS-noise } \\
(\mathrm{K})\end{array}$ \\
\hline${ }^{12} \mathrm{CO}$ & $J=1 \rightarrow 0$ & 115.271 & $\left(160^{\prime \prime} \times 160^{\prime \prime}\right)$ & 33 & 20 & 65 & 0.046 \\
${ }^{13} \mathrm{CO}$ & $J=1 \rightarrow 0$ & 110.204 & $\left(160^{\prime \prime} \times 160^{\prime \prime}\right)$ & 35 & 20 & 68 & 0.015 \\
$\mathrm{HCO}^{+}$ & $J=1 \rightarrow 0$ & 89.189 & $\left(140^{\prime \prime} \times 140^{\prime \prime}\right)$ & 43 & 20 & 87 & 0.020 \\
$\mathrm{H}^{13} \mathrm{CO}^{+}$ & $J=1 \rightarrow 0$ & 86.754 & $\left(180^{\prime \prime} \times 180^{\prime \prime}\right)$ & 44 & 20 & 84 & $0.005-0.1$ \\
\hline
\end{tabular}

the $\mathrm{HCO}^{+}$lines. This observation, as well as the line ratios of $\mathrm{HCO}^{+}$and $\mathrm{H}^{13} \mathrm{CO}^{+}$, shows that the former is optically thick in the line core and suggests that the observed dip is a self reversal effect, in agreement with the results of Hogerheijde et al. (1997). There is also a third peak or strong extended wing emission in some of the $\mathrm{HCO}^{+}$spectra which we identify with the outflow signature of L1551 IRS5.

In addition we also note the more puzzling presence of red-shifted material to the west of IRS5 where we find the blue-shifted wing (outflow) emission. There is also blue wing emission on the east side of IRS5, where the outflow component is present as red-shifted wing emission. This effect is also visible in the other molecular species studied by us (see e.g. Fig. 4). In Sect. 4.3 we comment on possible interpretations of this effect. In the top panel of Fig. 2 we display an overlay of the integrated line temperatures of the $\mathrm{H}^{13} \mathrm{CO}^{+}$for two different velocity ranges. This shows velocity variations over the inner regions of the L1551 IRS5 region. In this figure we can see a somewhat flattened structure with its major axis more or less perpendicular to the outflow axis. In addition, at least two discrete "clumps" can also be identified at positions of $+20^{\prime \prime},-60^{\prime \prime}$ and at $+60^{\prime \prime},+20^{\prime \prime}$ (see text below).

In the left bottom panel of Fig. 2a position-velocity diagram taken along an axis in the north-south direction of the top panel of that figure is shown. There is clearly a velocity gradient along this direction such that "redder" (more positive velocities) are found towards the north. A detailed analysis of the individual spectra reveals two emission peaks at different velocities. The "red", northern, velocity peak have an offset of $\left(-5^{\prime \prime},+20^{\prime \prime}, 6.4 \mathrm{~km} \mathrm{~s}^{-1}\right)$, while the "blue" velocity peak is found at offset $\left(0^{\prime \prime},-15^{\prime \prime}\right.$, $\left.+6.1 \mathrm{~km} \mathrm{~s}^{-1}\right)$. These peaks are found to be coincident with those found in the interferometric observations by Saito et al. (1996). When those data are convolved with our beam the agreement is perfect. The flattened structure can be traced in the position-velocity diagrams of Fig. 2 for at least $100^{\prime \prime}$, thus indicating a structure larger than what can be discerned from the interferometric observations which were not sensitive to structures larger than about 50". At this point we tentatively identify this flattened feature with a large (radius $\sim 7000$ AU) disklike structure surrounding both of the protostars. This is also consistent with the results of White et al. (2000), who based their modelling work on ISO spectroscopy of L1551 IRS5.

In the bottom right panel of Fig. 2 we display a position-velocity diagram taken along an axis between coordinates $+30^{\prime \prime},-80^{\prime \prime}$ and $-30^{\prime \prime},+80^{\prime \prime}$, and thus passing through the "southern clump" noted above. A comparison between the two bottom panels of Fig. 2 indicate that this "southern clump" is kinematically decoupled from the disk. This cut is along the plane of the tentative disk-like structure, and with a velocity gradient of $\sim 7.4 \pm 1.5 \mathrm{~km} \mathrm{~s}^{-1}(\mathrm{pc})^{-1}$ (disregarding the "southern clump"). This result is in excellent agreement with the early result of Kaifu et al. (1984). From our data it appears that the size of the disk is about $100^{\prime \prime}$ by $40^{\prime \prime}$. The disk structure is thus well resolved along the major axis.

We have further analysed the kinematic structure of the envelope by making position-velocity cuts along different directions. This has allowed us to infer a division between material belonging to the disk, and material assumed to form part of the surrounding envelope. We have produced similar diagrams at other orientations, and we find no obvious trace of the infall signature reported by Saito et al. (1996) in our $\mathrm{H}^{13} \mathrm{CO}^{+}$data. In a similar diagram of our $\mathrm{HCO}^{+}$data, however, the blueshifted signature identified with infall by Saito et al. is present at a velocity $\lesssim 5.5 \mathrm{kms}^{-1}$. We note here, however, that because of the geometry of the situation this material could also be due to outflow material spreading along the nearer surface of the outflow cone (see Sect. 4.3).

Inspection of Fig. 1 shows that the minimum emission (the self reversal) in $\mathrm{HCO}^{+}$is found at the same velocity (within errors) all over our map. It is thus probably representative of the envelope.

\subsection{The ${ }^{12} \mathrm{CO}$ and ${ }^{13} \mathrm{CO}$ data}

In Fig. 3 our new ${ }^{12} \mathrm{CO}$ and ${ }^{13} \mathrm{CO}$ spectra are displayed superposed on each other, at the same temperature scale. The signature of the outflow lobes are clearly visible. In Fig. 4 the spectra obtained in all transitions for the position $-20^{\prime \prime},+20^{\prime \prime}$ are displayed superposed on each other, demonstrating the velocity range of the outflow compared to the disk/envelope material as inferred from the $\mathrm{HCO}^{+}$and $\mathrm{H}^{13} \mathrm{CO}^{+}$spectra. 


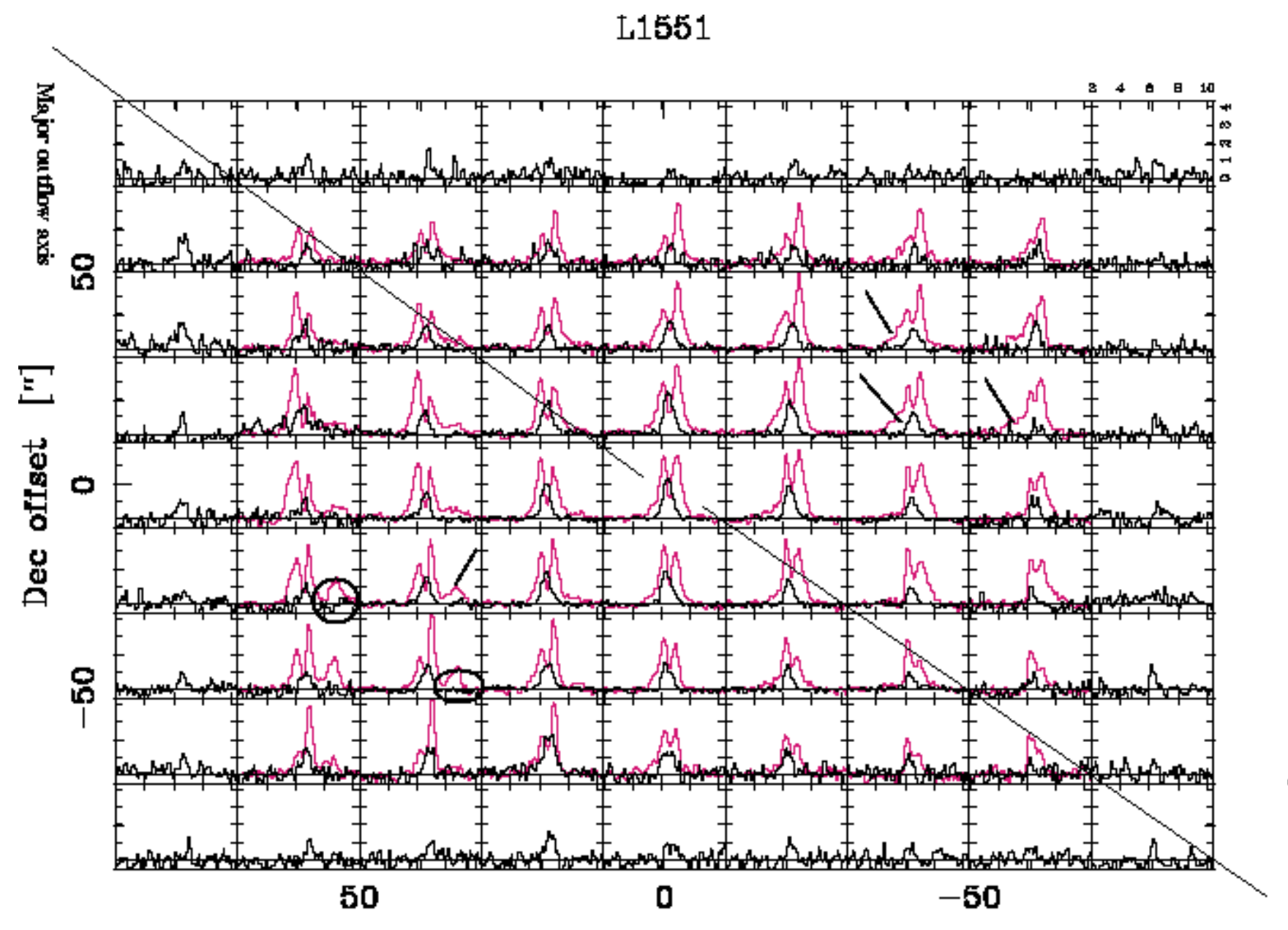

\section{RA offset ["]}

Fig. 1. Superposition of $\mathrm{H}^{13} \mathrm{CO}^{+}$and $\mathrm{HCO}^{+}$spectra. The $\mathrm{HCO}^{+}$spectra are displayed in red. Note the outflow signatures (the "third peak") towards the East (left, red-shifted wing emission) and West (right, blue-shifted wing emission). Some of the outflow signatures (which are only detectable in the $\mathrm{HCO}^{+}$spectra) have been marked by bold lines and circles. The position of the binary class I source L1551 IRS5 is at $0^{\prime \prime}, 0^{\prime \prime}$, and the major axis of the molecular outflow (and the optically visible jet) towards the lower right (SW) and the upper right (NE) and have also been marked by long bold lines.

\section{Discussion}

\subsection{The disk and envelope structure}

Our $\mathrm{H}^{13} \mathrm{CO}^{+}$data can be used to calculate the physical properties of the molecular gas close to L1551 IRS5. The results in Sect. 3 indicate that the $\mathrm{H}^{13} \mathrm{CO}^{+}$emission is optically thin. From Goldsmith et al. (1992) we may then write

$\frac{8 \pi k \nu^{2}}{h c^{3}} \int T_{\mathrm{R}}^{*} \mathrm{~d} v=A_{\mathrm{ul}} N_{\mathrm{u}} \Gamma$,

where the integral represents the antenna temperature of the line in question, corrected for atmospheric losses and coupling efficiency and integrated over velocity. $A_{\mathrm{ul}}$ is the Einstein coefficient for spontaneous emission and $\Gamma$ is a correction factor. $\Gamma$ depends on the excitation temperature, $T_{\mathrm{ex}}$, which relates the populations in the upper and lower levels of the transition and is given by

$\Gamma=1-\frac{\mathrm{e}^{h v / k T_{\mathrm{ex}}}-1}{\mathrm{e}^{h v / k T_{\mathrm{Bg}}}-1}$,

and is of order 1 for $T_{\mathrm{ex}} \gtrsim 6 \mathrm{~K}$ ( $T_{\mathrm{Bg}}$ is the temperature of the cosmic background radiation, $2.73 \mathrm{~K}$ ). The upper level column density, $N_{\mathrm{u}}$, is related to the total column density, $N$, through

$N_{\mathrm{u}}=f_{\mathrm{u}} N$,

where $f_{\mathrm{u}}$ is the fraction of the total number of molecules that are found in the upper level of the transition. Assuming a common excitation temperature for the lower 9 levels of the $\mathrm{H}^{13} \mathrm{CO}^{+}$molecule, we can derive $f_{\mathrm{u}}$ from the partition function (keeping in mind that this is only true under conditions of LTE, and otherwise likely to 


\section{$\mathrm{L} 1551 \mathrm{~N}$}

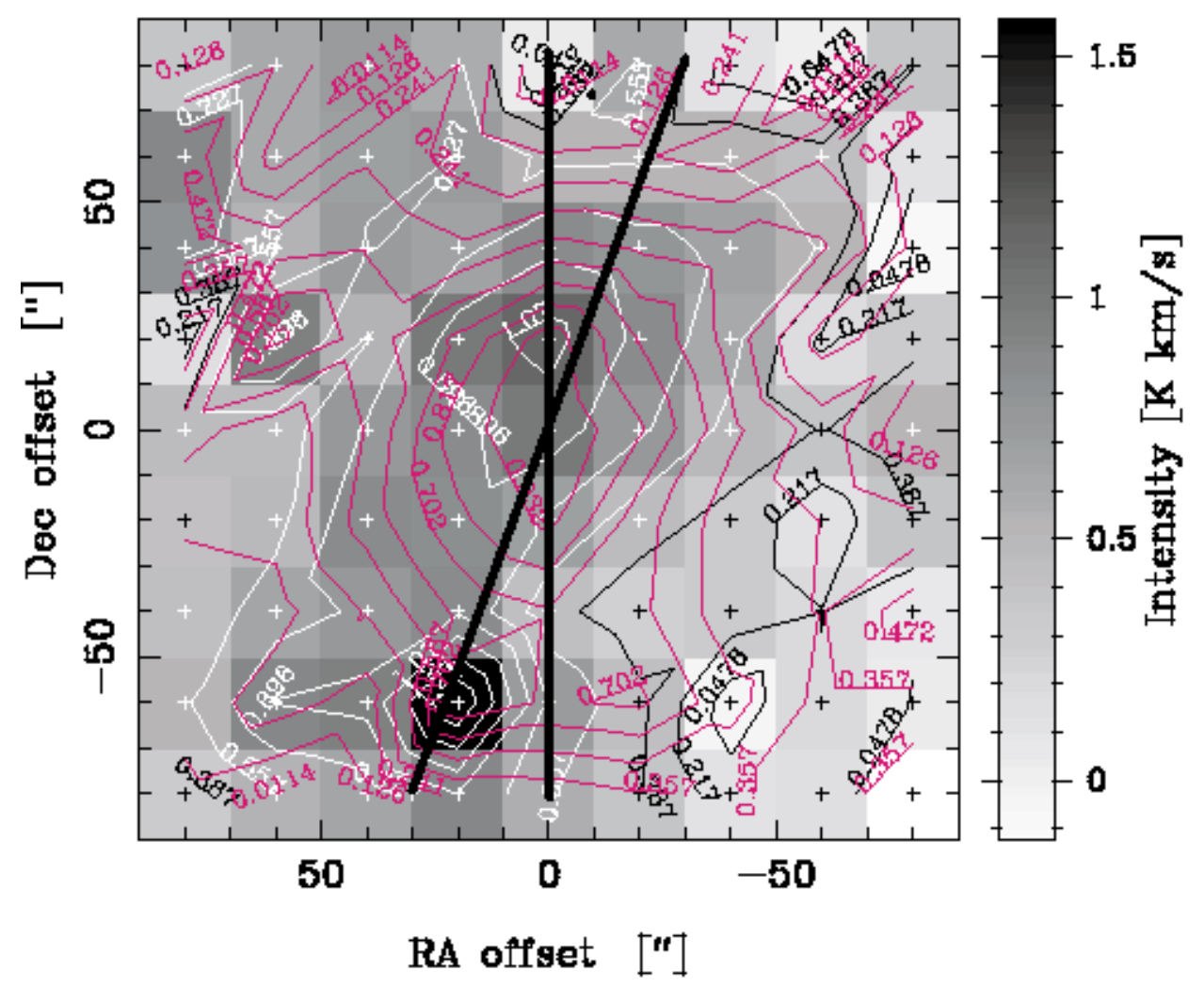

L1551

L1551
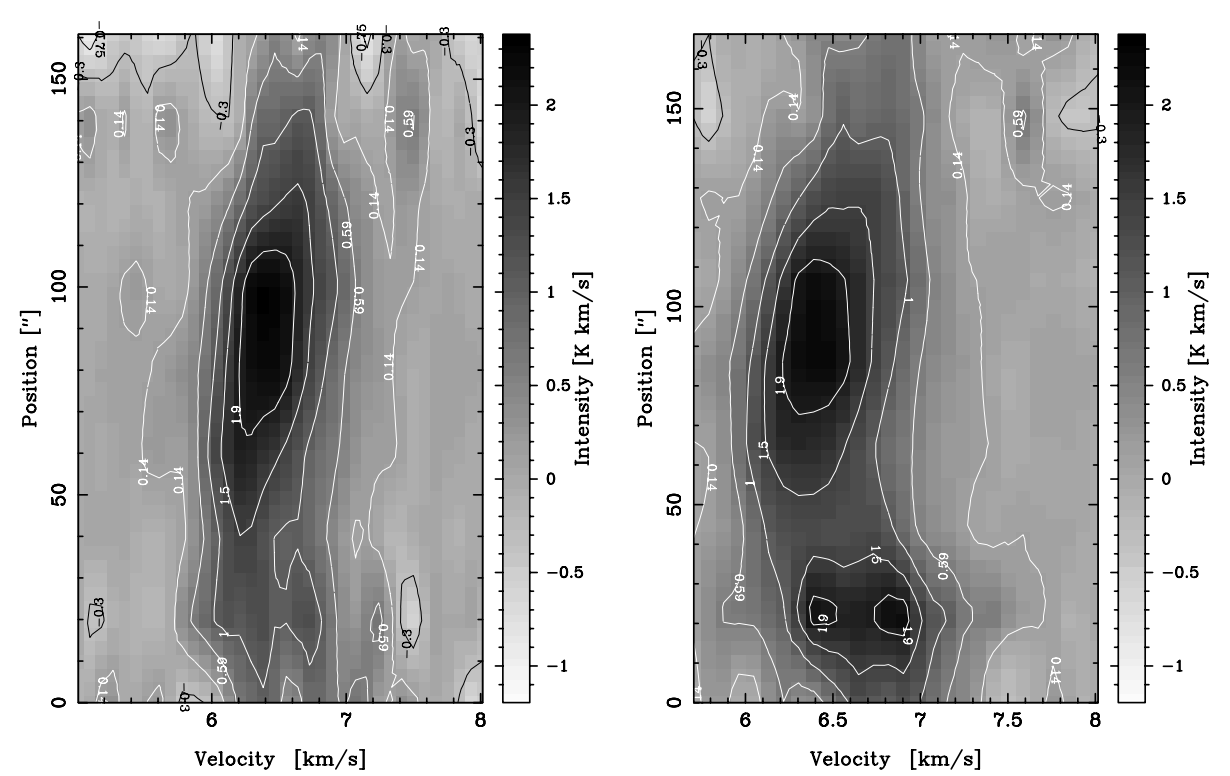

Fig. 2. (Top) The integrated $\mathrm{H}^{13} \mathrm{CO}^{+}$line emission between $6.5 \mathrm{~km} \mathrm{~s}^{-1}$ and $7.4 \mathrm{~km} \mathrm{~s}^{-1}$ (white contours and grey scale) overlayed with the contours for the $\mathrm{H}^{13} \mathrm{CO}^{+}$emission integrated between $6.0 \mathrm{~km} \mathrm{~s}^{-1}$ and $6.5 \mathrm{~km} \mathrm{~s}^{-1}$ (red). The major axis of the molecular outflow is from the upper left corner through the position of L1551 IRS5 $\left(0^{\prime \prime}, 0^{\prime \prime}\right)$, towards the lower right corner. The orientation along which the two cuts in the bottom panels of the figure have been taken are marked in this panel. Note the flattened contours along a direction orthogonal to the outflow axis. Also note the two distinct "clumps" at positions $\left(+20^{\prime \prime},-60^{\prime \prime}\right)$ and $\left(+60^{\prime \prime}\right.$, $\left.+20^{\prime \prime}\right)$. (Bottom left) Velocity Intensity plot of the $\mathrm{H}^{13} \mathrm{CO}^{+}$line emission between $5.0 \mathrm{~km} \mathrm{~s}^{-1}$ and $8.0 \mathrm{~km} \mathrm{~s}^{-1}$. Contours are in $\mathrm{K} \mathrm{km} \mathrm{s}^{-1}$. The orientation is along the cut through our map in the north - south direction with north towards the top. (Bottom right) A cut through our map from position $+30^{\prime \prime},-80^{\prime \prime}$ towards $-30^{\prime \prime},+80^{\prime \prime}$, and passing through the "south clump". Again, North is towards the top. The velocity resolution is $86 \mathrm{~m} \mathrm{~s}^{-1}$ in both panels, and IRS5 is found at position-coordinate $\sim 85^{\prime \prime}$. See top panel for a display of the cuts along which the velocity-intensity plots have been made. Note the slightly different velocity scale in the right panel. 


\section{L1551}

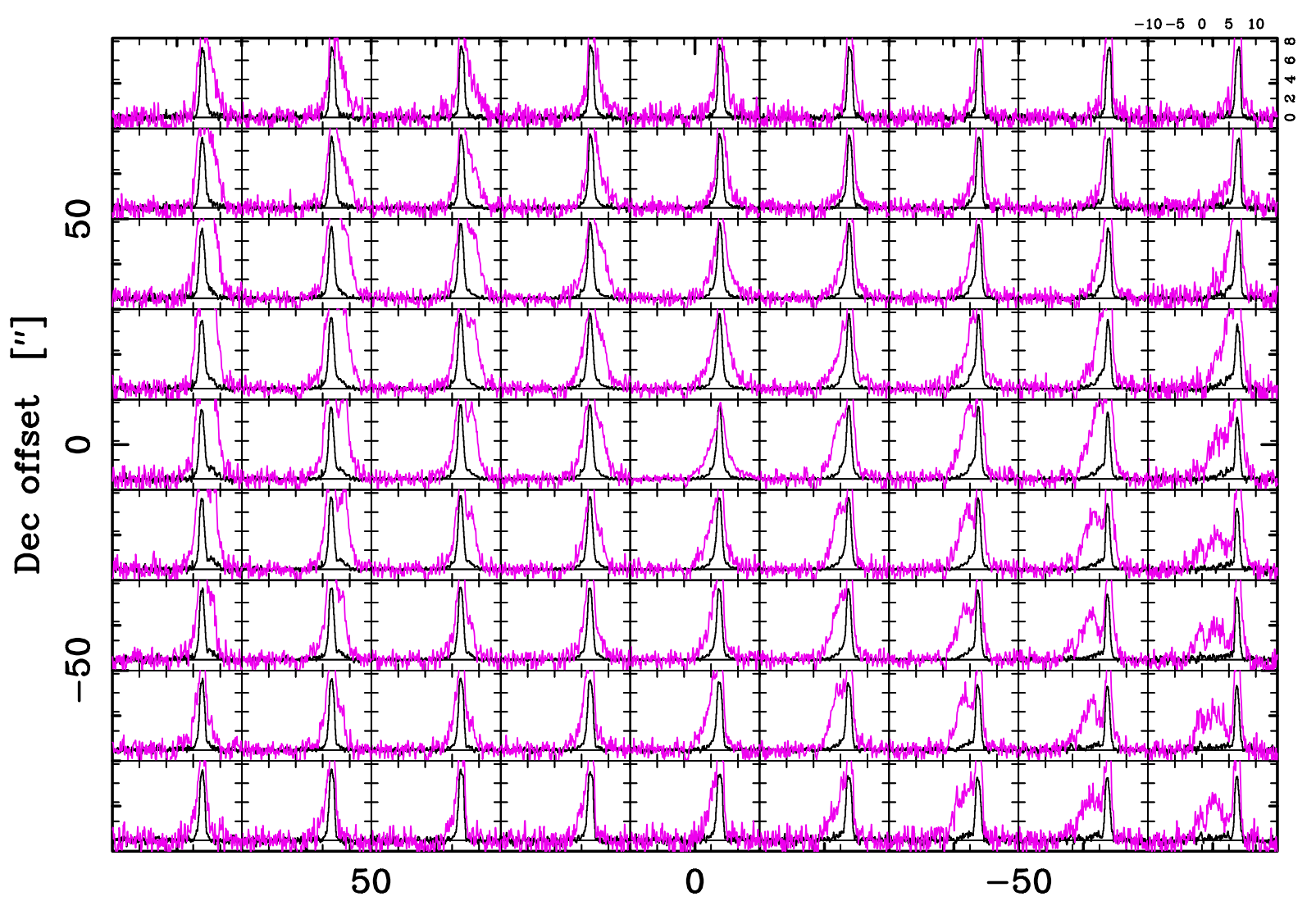

RA offset ["]

Fig. 3. Superposition of the ${ }^{13} \mathrm{CO}$ and the ${ }^{12} \mathrm{CO}$ spectra. The ${ }^{12} \mathrm{CO}$ are shown in red. Note the wing emission on both sides of the central peak at positions of RA offset $0^{\prime \prime}$ and progressing to the maps limits both north and south.

overestimate the value of the partition function. Note that an overestimation leads to an increased mass)

$Q(T)=\sum_{i=1}^{9} g_{i} \mathrm{e}^{\frac{-E_{i}}{k T}}$

where $g_{i}$ is the statistical weight for the $i$ th level. This will give us the upper level fraction for the 1-0 transition through

$f_{\mathrm{u}(1-0)}=g_{1} \frac{\mathrm{e}^{\frac{-E_{i}}{k T}}}{Q(T)}$.

In order to apply this formalism to our result, we also have to assume a temperature structure through the disk and envelope surrounding the IRS5 binary. Following Emerson (1988), and Terebey et al. (1993), we may write:

$T_{\mathrm{D}}(r) \approx T_{0}\left(\frac{r}{r_{0}}\right)^{-q}\left(\frac{L_{*}}{L_{0}}\right)^{q / 2}$,

for the dust temperature $T_{\mathrm{D}}(r)$ as a function of distance from the protostar. (We are implicitly assuming that the dust is optically thin with respect to the infrared radiation, and that the dust and the molecular gas is in equlibrium.) Here $q=2 /(4+\beta)$ and depends on the actual dust properties; $\beta$ is the emissivity, generally assumed to be between 1 and 2 . We then have:

$T_{\text {dust }}\left(r, L_{*}\right) \approx 38 \mathrm{~K}\left(\frac{r}{100 \mathrm{AU}}\right)^{-0.4}\left(\frac{L_{*}}{1 L_{\odot}}\right)^{0.2}$,

with $L_{*}$ equal to $28 L_{\odot}$ (see Motte \& André 2001) for the IRS5 binary.

Using this simple 2-dimensional temperature model we have calculated the mass of the molecular envelope surrounding L1551 IRS5. The result is $\sim 4.4 M_{\odot}$ of molecular material, assuming that the abundance ratio $\left[\mathrm{H}^{13} \mathrm{CO}^{+}\right] /\left[\mathrm{H}_{2}\right]$ is $5.5 \times 10^{-11}$ (Butner et al. 1995). The accuracy of this result depends on how sensitive the solution is with respect to the assumptions we made. Under the given assumptions, $0.15 \lesssim f_{\mathrm{u}} \lesssim 0.5$. It is however, only for temperatures $\gtrsim 20 \mathrm{~K}$ that significant deviations of $f_{\mathrm{u}}$ will occur and only the innermost portion of our central beam $\left(r \lesssim 10^{\prime \prime}\right)$ will be important in this respect. The solution for the column density is then relatively insensitive to the hypothesis of a constant excitation temperature along each line of sight (the third dimension). Assumning a constant $T_{\mathrm{ex}} \sim 10 \mathrm{~K}$ in the complete envelope and disk lowers the total mass with only $\sim 20 \%$. Our mass estimate is sensitive, however, to the assumed abundance of 


\section{L1551}

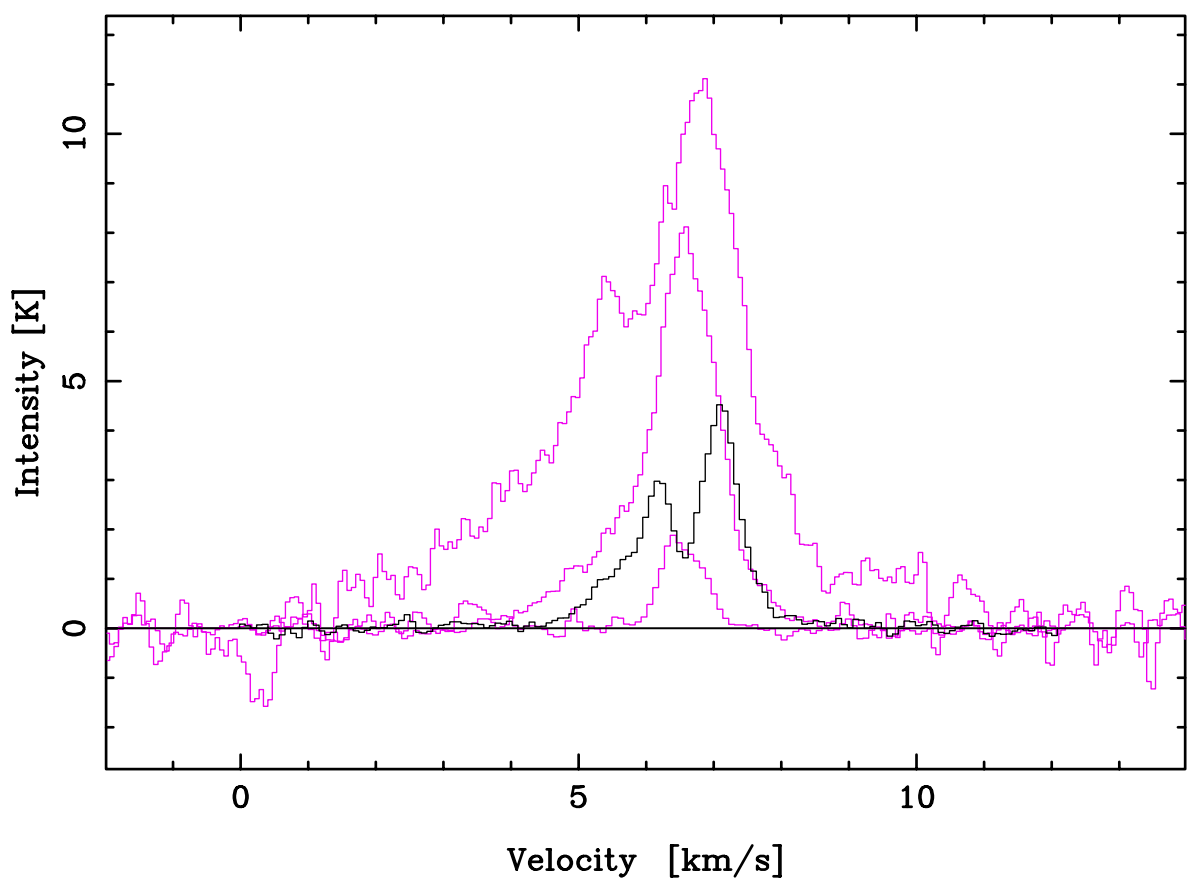

Fig. 4. Superposition of all transitions obtained towards the position $-20^{\prime \prime},+20^{\prime \prime}$. The outermost controur is that of ${ }^{12} \mathrm{CO}$, followed by ${ }^{13} \mathrm{CO}, \mathrm{HCO}^{+}$(in black) and innermost $\mathrm{H}^{13} \mathrm{CO}^{+}$. All intensities are in units of $T_{\mathrm{R}}^{*}$.

$\mathrm{H}^{13} \mathrm{CO}^{+}$, as well as to the actual value of the dipole moment. Following Butner et al. (1995) we have assumed a dipole moment of 4.07 Debye noting that using the lower 'standard' value of 3.3 Debye (Pickett et al. 1998) will increase the mass estimate with $\sim 50 \%$. For the value of the abundance $X\left[\mathrm{H}^{13} \mathrm{CO}^{+}\right]$Hogerheijde et al. (1997) have found about $1.7 \times 10^{-10}$ for a sample of dense cores in Taurus. Pratap et al. (1997) find similar values. On the other hand, Butner et al. (1995) determine $5.5 \times 10^{-11}$ for L1551 itself, and about $1.0 \times 10^{-10}$ for a number of similar sources.

If we disregard the kinematically decoupled material (i.e. the clump mentioned in Sect. 3), as well as the clump found within the "red" outflow lobe (see Fig. 2), we can arrive at a tentative disk mass. We find that the disk contain $\sim(2.5 \pm 1.5) M_{\odot}$ of molecular material (with the higher value representing the lowest values of $X\left[\mathrm{H}^{13} \mathrm{CO}^{+}\right]$) and the dipole moment, while the two clumps contain $\sim(0.65 \pm 0.25) M_{\odot}$ and $\sim(0.35 \pm 0.15) M_{\odot}$ respectively. These results are in good agreement with the original suggestions by Kaifu et al. (1984), which were based on single dish CS observations.

We have also calculated the radiative transfer of $\mathrm{HCO}^{+}$ and $\mathrm{H}^{13} \mathrm{CO}^{+}$of a model approximating the region surrounding L1551 IRS5. The solution is reached by means of accelerated lambda iteration (e.g. Rybicki \& Hummer 1991) for a spherical core-envelope model, assuming the following parameters:

- Core radius $=5000 \mathrm{AU}$;

- Envelope radius $=12500 \mathrm{AU}$;
- Constant abundances are kept for both $\mathrm{HCO}^{+}$and $\mathrm{H}^{13} \mathrm{CO}^{+}$throughout the model;

- The turbulent velocity $V_{\text {turb }}$, of $0.45 \mathrm{kms}^{-1}$ is set to match the observed FWHM of the $\mathrm{H}^{13} \mathrm{CO}^{+}$line $\left(0.8 \mathrm{~km} \mathrm{~s}^{-1}\right)$;

- The spectra have been calculated for beam sizes $44^{\prime \prime}$ $\left(\mathrm{H}^{13} \mathrm{CO}^{+}\right)$and $43^{\prime \prime}\left(\mathrm{HCO}^{+}\right)$with unity beam filling factor.

The resulting model is summarised in Table 3. While our model is a simplification (e.g. simplified geometry, beam filling of one, constant abundances), it should at least provide qualitative agreement. Note that $T_{\text {kin }}$ (in the core) could be somewhat higher if the source is clumpy. For a beam filling factor of $2 / 3, T_{\text {kin }}$ could be set to $15 \mathrm{~K}$ in the core (and $10 \mathrm{~K}$ in the envelope) without affecting the line shapes too much. The $\mathrm{H}_{2}$ density in the envelope is - on the other hand - quite critical. It must be $0.8 \times 10^{4} \mathrm{~cm}^{-3} \lesssim$ $n\left[\mathrm{H}_{2}\right] \lesssim 3.0 \times 10^{4} \mathrm{~cm}^{-3}$ if the self-absorption is to be as strong as is actually observed. A sample of the calculated model spectra are displayed in Fig. 5. The model is indeed in good agreement with the observations both qualitatively and quantitatively given the uncertainties.

The molecular gas observed in the vicinity of L1551 IRS5 thus appears to consist of four components:

1. An elongated dense structure - best traced in $\mathrm{H}^{13} \mathrm{CO}^{+}$;

2. An envelope - outlined by the self-reversed $\mathrm{HCO}^{+}$;

3 . Wing emission in the central part of the $\mathrm{HCO}^{+}$profile - including the so called anomalous emission (see Sect. 4.3; 
Table 3. The radiative transfer model parameters and results.

\begin{tabular}{lrrrrrr}
\hline & $\begin{array}{r}T_{\text {kin }} \\
(\mathrm{K})\end{array}$ & $\begin{array}{r}n[\mathrm{H} 2] \\
\left(\mathrm{cm}^{-3}\right)\end{array}$ & $\begin{array}{r}M \\
\left(M_{\odot}\right)\end{array}$ & $\begin{array}{r}V_{\text {turb }} \\
\left(\mathrm{km} \mathrm{s}^{-1}\right)\end{array}$ & $X[\mathrm{HCO}+]$ & $X[\mathrm{H} 13 \mathrm{CO}+]$ \\
\hline Core: & 10 & $3.2 \times 10^{5}$ & 0.96 & 0.45 & $2.0 \times 10^{-9}$ & $5.5 \times 10^{-11}$ \\
Envelope: & 6 & $1.0 \times 10^{4}$ & 0.43 & 0.45 & $2.0 \times 10^{-9}$ & $5.5 \times 10^{-11}$ \\
\hline
\end{tabular}

\section{L1551 ALI model}

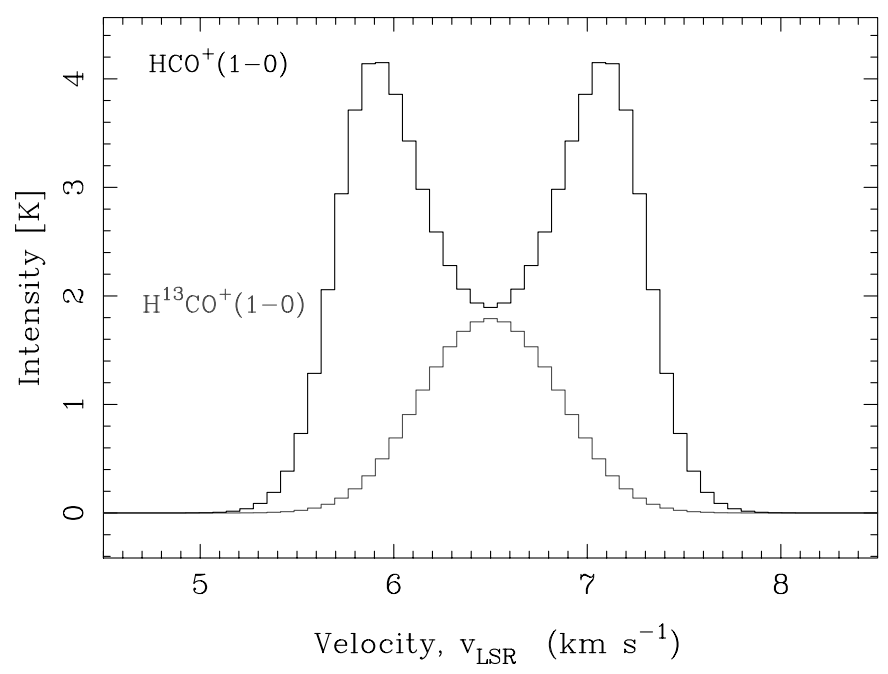

Fig. 5. Representative calculated model spectrum for $\mathrm{HCO}^{+}$ (outer spectrum) and $\mathrm{H}^{13} \mathrm{CO}^{+}$(inner spectrum). The position is the one of L1551 IRS5 $\left(0^{\prime \prime}, 0^{\prime \prime}\right)$. All intensities are in units of $T_{\mathrm{R}}^{*}$.

4. The outflow - seen in $\mathrm{HCO}^{+},{ }^{12} \mathrm{CO}$ and ${ }^{13} \mathrm{CO}$, at higher relative velocities (see e.g. Fig. 4).

One explanation for the flattened structure seen in the $\mathrm{H}^{13} \mathrm{CO}^{+}$and $\mathrm{HCO}^{+}$data, taking also the associated velocity gradient into account, is a rotating dense molecular disk.

Two optical jets have been identified by Fridlund \& Liseau (1998) to be emanating from behind the obscuration of IRS5. These authors find it likely that each of the stellar components is the source of one of these jets (for reasons of morphology and from the velocity field of the jets). The major axis of these two jets are in the same general direction, which is also that of the major axis of the molecular outflow. The symmetry axis of the jets also roughly define what the orientation of the accretion disk plane will be for both stellar components (approximately orthogonal to the major axis of the jets). This is also the major axis of the flattened structure. We thus conclude that a likely explanation for the structure observed by us in $\mathrm{HCO}^{+}$and $\mathrm{H}^{13} \mathrm{CO}^{+}$is a massive $\left(\sim 1-4 M_{\odot}\right)$ molecular disk rotating around the protostellar binary L1551 IRS5. This disk, with a size scale of $\sim 7000$ AU, is thus likely to be both rotationally and magnetically supported. It is important in this context to understand that structures at these scales will have different properties from the much smaller (<100 AU- 1000 AU) structures that are directly accreting onto the protostar(s), and that therefore the term "disk" will have to be used with this in mind.

\subsection{The outflow signatures near IRS5}

With our data, we can make an improved determination of the outflow mass and energetics in the inner two arcminutes of the molecular outflow. The line intensity wing ratio $I\left({ }^{13} \mathrm{CO}\right) / I\left({ }^{12} \mathrm{CO}\right)$ is much less than what would be expected if both lines where optically thin in the wing (see e.g. Fig. 4). We therefore assume that at least ${ }^{12} \mathrm{CO}$ is optically thick. If we ignore the $2.7 \mathrm{~K}$ background radiation we have:

$\frac{T_{\mathrm{R}}^{*}(\nu)}{b_{\mathrm{f}}}=\left[\frac{h \nu / k}{\mathrm{e}^{h \nu / k T_{\mathrm{ex}}-1}}\right]\left(1-\mathrm{e}^{-\tau_{\nu}}\right)$.

Assuming the same $T_{\text {ex }}$ as in the model described in the previous subsection, we can calculate the molecular outflow mass. This will be a minimum mass because of the beam filling factor, $b_{\mathrm{f}}$, which is set equal to unity in these calculations. We determine a value of $0.4 M_{\odot}$ at velocities between $+3 \mathrm{kms}^{-1}$ and $+6 \mathrm{~km} \mathrm{~s}^{-1}$, while between $+7 \mathrm{~km} \mathrm{~s}^{-1}$ and $+10 \mathrm{~km} \mathrm{~s}^{-1}$ we obtain $0.5 M_{\odot}$. Completing the same exercise for $\mathrm{HCO}^{+}$we arrive at $\sim 0.15 M_{\odot}$ in each velocity interval. Taking the inclination into account, this translates to a mass loss of $\dot{M} \sim 10^{-5} M_{\odot} \mathrm{yr}^{-1}$ $\sim 5 \times 10^{-5} M_{\odot} \mathrm{yr}^{-1}$. These values are in good agreement with the results of Fridlund \& Knee (1993) and Hogerheijde et al. (1998).

\subsection{Infall or outflow? - the anomalous "red" and "blue" emission signatures}

As mentioned previously, there is a significant amount of wing emission found on the "wrong" side of the line core in several transitions. This has been commented on by several authors - using both single dish and interferometric observations (see below). The available data suggest two possibilities:

1. It is assumed that the major axis of the outflow in L1551 is oriented at a small angle with respect to the plane of the sky (Liseau \& Sandell 1986), and that the outflow has the shape of bipolar cones of sweptup shells of molecular gas (Fridlund et al. 1989) - the cones also being filled with some molecular gas. As we view the outflow essentially from the side, the anomalous wings could then be caused by emission emanating from the far side of the outflow cone (relative to the major part of the gas on either side of L1551 IRS5) and 


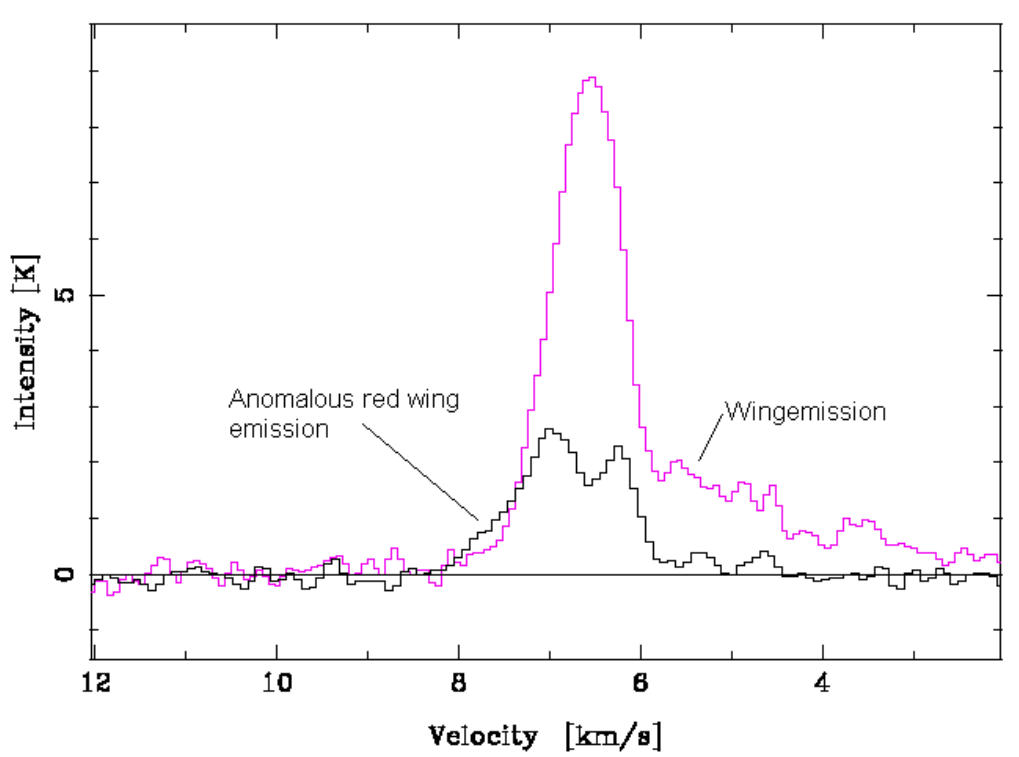

Fig. 6. The anomalous wing emission demonstrated in ${ }^{13} \mathrm{CO}$ and $\mathrm{HCO}^{+}$. The data in this figure is from the position offset $0^{\prime \prime},-60^{\prime \prime}$.

due to the geometry of the situation (with the outflow axis emerging out of the plane of the sky at a relatively shallow angle), thus receeding from or approaching the Earth (Fridlund et al. 1989).

2. These anomalous signatures could be due to infall of molecular gas towards the surface of the disk-like structure as has been suggested by Saito et al. (1996), Ohashi et al. (1996) and Momose et al. (1998).

We favour the first of these options for the following reasons: Originally, Kaifu (1987) and Uchida et al. (1987) interpreted this effect as signs of rotation in the outflow lobes, such as predicted by the Uchida-Shibata theory (Uchida \& Shibata 1984). A similar effect was also reported by Liljeström (1989). Fridlund (1987) and Fridlund et al. (1989) noted that since this behaviour is present all across the extent of the outflow (more than $10^{5} \mathrm{AU}$ ), it is not likely that it is due to infall. Further, referring to Fig. 6 we see that the anomalous wing emission tend to be stronger in $\mathrm{HCO}^{+}$than in ${ }^{13} \mathrm{CO}$. This is the case in general over our map, and indicates that the gas responsible for the anomalous wing tends to be very dense. This is exactly what would be expected if we were observing a dense swept up shell from the side.

\section{Conclusions}

1. The $\mathrm{HCO}^{+}$gas detected in the close vicinity of IRS5, is found in our observations to possess an optically thick core with a self-reversal in the line core over much of our map. The strength of the self-reversal is at a maximum along a line roughly orthogonal to the major axis of the molecular outflow. We interpret this as defining the disk plane.
2. The $\mathrm{H}^{13} \mathrm{CO}^{+}$line profile is roughly gaussian with a slight assymetry that appears as a velocity gradient of $7.4 \pm 1.5 \mathrm{~km} \mathrm{~s}^{-1}(\mathrm{pc})^{-1}$. The line peak is found close to the velocity of the self reversal seen in the $\mathrm{HCO}^{+}$ transition. Our data indicate that $\mathrm{H}^{13} \mathrm{CO}^{+}$is optically thin, or nearly so, and we use it to calculate the mass of the disk and its associated envelope.

3. The mass of the molecular envelope and disk is found, from $\mathrm{H}^{13} \mathrm{CO}^{+}$, to be $\sim(2.5 \pm 1.5) M_{\odot}$, strongly supporting the original interpretation of Kaifu et al. (1984) CS observations.

4. Two isolated clumps with masses $\sim 0.5 M_{\odot}-\sim 1 M_{\odot}$ are found in the vicinity of L1551 IRS5. They both appear kinematically unconnected to the disk/envelope structure, and in one case is (for morphological reasons) believed not connected with the outflow either.

5. The line wings of ${ }^{13} \mathrm{CO}$ and $\mathrm{HCO}^{+}$are optically thin and we use this to calculate the outflow mass in the two transitions. We find a value of $0.9 M_{\odot}$ for ${ }^{13} \mathrm{CO}$ and $\sim 0.3 M_{\odot}$ for $\mathrm{HCO}^{+}$. This is the sum of the molecular gas in both the red- and the blue-shifted gas.

6. The outflow mass loss rate determined from our data is found to be $\dot{M} \sim 10^{-5} M_{\odot} \mathrm{yr}^{-1} \sim 5 \times 10^{-5} M_{\odot} \mathrm{yr}^{-1}$, confirming the result of Fridlund \& Knee (1993) that L1551 IRS5 has a high rate of outflow.

7. The anomalous wing emission found near IRS5 is interpreted as being due to emission emanating from the far side of the outflow lobes and not likely to be caused by infall.

Acknowledgements. We are grateful to the staff at Onsala Space Observatory (OSO), in particular to Dr. L. E. B. Johansson for help during the observations. The OSO $20 \mathrm{~m}$ telescope is operated by the Swedish National Facility for Radio Astronomy, Onsala Space Observatory at Chalmers 
University of Technology. We also acknowledge fruitful discussions with Drs. G. Olofsson, P. Papadopoulos and R. Liseau. Finally, we acknowledge the constructive comments of the referee, Dr G. A. Blake, which resulted in improvements to the paper.

\section{References}

Bieging, J. H., \& Cohen, M. 1985, ApJL, 289, L5

Butner, H. M., Lada, E. A., \& Loren, R. B. 1995, ApJ, 448, $207+$

Emerson, J. P. 1988, in NATO ASIC Proc. 241, Formation and Evolution of Low Mass Stars, 21+

Fridlund, C. 1987, Ph.D. Thesis, University of Stockholm, Sweden

Fridlund, C. V. M., \& Knee, L. B. G. 1993, A\&A, 268, 245

Fridlund, C. V. M., \& Liseau, R. 1998, ApJL, 499, L75

Fridlund, C. V. M., Nordh, H. L., van Duinen, R. J., Aalders, J. W. G., \& Sargent, A. I. 1980, A\&A, 91, L1

Fridlund, C. V. M., Sandqvist, A., Nordh, H. L., \& Olofsson, G. 1989, A\&A, 213, 310

Fridlund, M., Huldtgren, M., \& Liseau, R. 1997, in IAU Symp. 182, Herbig-Haro Flows and the Birth of Stars, 19

Fuller, G. A., Ladd, E. F., Padman, R., Myers, P. C., \& Adams, F. C. 1995 , ApJ, 454, 862+

Goldsmith, P. F., Margulis, M., Snell, R. L., \& Fukui, Y. 1992, ApJ, 385, 522

Hogerheijde, M. R., van Dishoeck, E. F., Blake, G. A., \& van Langevelde, H. J. 1997, ApJ, 489, 293+

Hogerheijde, M. R., van Dishoeck, E. F., Blake, G. A., \& van Langevelde, H. J. 1998, ApJ, 502, 315+

Kaifu, N. 1987, in Star Forming Regions, IAU Symp., 115, 275

Kaifu, N., Hasegawa, T., Morimoto, M., et al. 1984, A\&A, 134,7

Keene, J., \& Masson, C. R. 1990, ApJ, 355, 635

Kutner, M. L., \& Ulich, B. L. 1981, ApJ, 250, 341

Ladd, E. F., Fuller, G. A., Padman, R., Myers, P. C., \& Adams, F. C. 1995 , ApJ, 439, 771

Lay, O. P., Carlstrom, J. E., Hills, R. E., \& Phillips, T. G. 1994, ApJL, 434, L75

Liljeström, T. 1989, A\&A, 219, L19

Liseau, R., \& Sandell, G. 1986, ApJ, 304, 459
Looney, L. W., Mundy, L. G., \& Welch, W. J. 1997, ApJL, 484, L157

Mizuno, A., Onishi, T., Hayashi, M., et al. 1994, Nature, 368, $719+$

Momose, M., Ohashi, N., Kawabe, R., Nakano, T., \& Hayashi, M. 1998, ApJ, 504, 314+

Moneti, A., Forrest, W. J., Pipher, J. L., \& Woodward, C. E. 1988, ApJ, 327, 870

Motte, F., \& André, P. 2001, A\&A, 365, 440

Ohashi, N., Hayashi, M., Ho, P. T. P., Momose, M., \& Hirano, N. 1996, ApJ, 466, 957+

Pickett, H. M., Poynter, R. L., Cohen, E. A., et al. 1998, J. Quant. Spectrosc. Rad. Transfer, 60, 883+

Pratap, P., Dickens, J. E., Snell, R. L., et al. 1997, ApJ, 486, $862+$

Rainey, R., White, G. J., Richardson, K. J., et al. 1987, A\&A, 179, 237

Reipurth, B. 1999, A general catalogue of Herbig-Haro objects, electronically published via World Wide Web at http: casa.colorado.edu/hhcat

Rodriguez, L. F., Canto, J., Torrelles, J. M., \& Ho, P. T. P. 1986, ApJL, 301, L25

Rodriguez, L. F., D'Alessio, P., Wilner, D. J., et al. 1998, Nature, 395, 355

Rybicki, G. B., \& Hummer, D. G. 1991, A\&A, 245, 171

Saito, M., Kawabe, R., Kitamura, Y., \& Sunada, K. 1996, ApJ, $473,464+$

Sargent, A. I., Keene, J., Masson, C., \& Beckwith, S. 1988, ApJ, 333, 936

Smith, H. A., Fischer, J., Schwartz, P. R., \& Geballe, T. R. 1987, ApJ, 316, 265

Snell, R. L., Loren, R. B., \& Plambeck, R. L. 1980, ApJL, 239, L17

Stocke, J. T., Hartigan, P. M., Strom, S. E., et al. 1988, ApJS 68, 229

Terebey, S., Chandler, C. J., \& Andre, P. 1993, ApJ, 414, 759

Uchida, Y., Kaifu, N., Hayashi, S. S., Hasegawa, T., \& Shibata, K. 1987, in Star Forming Regions, IAU Symp., 115, 287

Uchida, Y., \& Shibata, K. 1984, PASJ, 36, 105

White, G. J., Liseau, R., Men'shchikov, A. B., et al. 2000, A\&A, 364, 741

Woody, D. P., Scott, S. L., Scoville, N. Z., et al. 1989, ApJ, 337, L41 